\title{
A Multi-Centers Comparative Study of Extensive and Deep Degree Burns of Children
}

Yongchong Chen ${ }^{1}$, Guiru $\mathrm{Li}^{2}$, Wardhana Aditya ${ }^{3}$, Renliang $\mathrm{He}^{4}$, Gleen Angelo S. Genuino ${ }^{5}$, Zijun $\mathrm{Fu}^{6}$, Roys A. Pangayoman ${ }^{7}$ and Xiangning Wang ${ }^{6}$

1. Department of Burns and Plastic Surgery of Beijing NanYuan Hospital, Feng Tai District, Beijing 100076, China

2. Department of Surgery of Lugouqiao Hospital, Feng Tai District, Beijing 100165, China

3. Rumah sakit Islam Pusat Jakarta (Islamic Central Hospital Jakarta) and Plastic and Burns Surgery Division, Cipto Mangunkusumo Hospital of University Indonesia, Jl. Diponegoro No. 71 Jakarta 10430, Indonesia

4. Department of Burns, Wounds and Ulcers of the People's Hospital of Zhongshan, Huangpu Guangdong 528429, China

5. Department of Burns and Plastic Surgery of PGH, Manila Philippines

6. Department of Burns, Wounds and Ulcers of Ya'an People's Hospital, Sichuan 625000, China

7. R.S Immanuel Surgery Department, Jl Kopo 161 Bandung, West Java 40146, Indonesia

\begin{abstract}
Objective: To summarize and assess the therapeutic effects of Burns Regenerative Medicine and Therapy (BRT) and Eschar Excision and Skin Grafting Techniques (SGT) on extensive and deep degree pediatric burns treatment. Methods: A multi-center controllable randomization comparative method was used in this study. The 69 pediatric in-patients with extensive and deep degree burns that from 7 centers in 3 countries were separate randomization into test group ( 37 cases were treated with BRT) and control group ( 32 cases were treated with SGT). The main outcome measures included average usage rates of analgesic in treatment, wound infection rates, skin grafting rates, average wound healing time, scar hyperplasia rates after treatment, dysfunction rates and mortality. Statistical analysis was applied to the data of both groups. Results: No significant difference was found in mortality between test group (2.70\%) and control group (3.13\%); The results of t-test or $\mathrm{x}^{2}$-test of other main outcome measures showed significant difference $(\mathrm{P}<0.01)$ between the two groups. Conclusion: Burns regenerative medicine and therapy provides a more satisfactory treatment for extensive and deep degree pediatric burns.
\end{abstract}

Key words: Pediatric burns, extensive and deep degree burns, burns regenerative medicine and therapy, moist exposed burns ointment (MEBO), eschar excision, skin grafting technique.

\section{Introduction}

Incidence of pediatric burns is rather high due to children's weak ability to self control, response fast, coordinate and foresee the danger. Pediatric burns form $32.82 \%$ of the hospitalized burns and should not be ignored [1]. Especially when the total burns area is more than 25\% TBSA (Extensive burn), in case of unsuitable treatment, it is very easy to result in hypertrophic scar, contracture, disfigure and lifelong

Corresponding author: Yongchong Chen, $\mathrm{MD}, \mathrm{PhD}$, research fields: burns regenerative medicine and therapy. Email: chenyc@chinaburn.com. disability. These serious consequences make it very difficult for the children patients to recover physiological function and mental health, reintegrate into family, school and society. In order to find an ideal method to treat extensive and deep degree pediatric burns, we treated the severe extensive and deep degree pediatric burns with BRT or SGT in 7 centers since October 1996 to October 2005. After the analysis and comparative study, we found that the application of BRT in treating extensive and deep degree pediatric burns can achieve a more satisfactory clinical outcome. The summarized report is as below. 


\section{Clinical Data}

\subsection{Criteria of Inclusion}

(1) The hospitalized children with burn injuries from October 1996 to October 2005; (2) The age of patients: from 1year to 12 years old; (3) Clinical diagnosis at admission: burns area $>$ or $=25 \%$ TBSA and the area of deep degree burns (Deep II or III degree) $>10 \%$ TBSA;

(4) Reasons of burns: thermal burn (flame, hot liquid, etc) and chemical burn (acids, alkali, etc); (5) Treated with BRT or SGT.

\subsection{Exclusion Criteria}

(1) Dysplastic premature or undernourished children; (2) Children under 1 year or over 12 years old, (3) Children have congenital disease or severe primary disease before injured; (4) Electrical burns and radiation burns.

\subsection{Diagnostic Criteria}

The degrees of burns are diagnosed uniformly according to the criteria in the Blue Book of Burns Regenerative Medicine [2]. Deep second degree burn means full-thickness skin necrosis, including epidermis and dermis are injured; Third degree burn means the injury reaches to the subcutaneous tissues and beyond. Deep burns in this study include deep second degree and third degree burn injuries. The calculation of burns area is in accordance with the active Chinese standard of classification of severity of pediatric burns [3]. The 'hand method' will be used to determine the area of burn (i.e., the palmar surface area covered by the patient's open hand without left space between fingers, which is roughly $1 \%$ of the whole body surface.), and/or in combination with the formula to calculate the burns area: TBSA $\%$ of head and neck $=9+(12-$ Age $)$, $\mathrm{TBSA} \%$ of lower part $=46-(12-$ Age $)$.

\subsection{Data of Test Group (A Group)}

There were 37 cases with extensive and deep degree pediatric burns were treated by BRT and met the inclusion criteria. 24 males and 13 females, the age from 1 year and 6 months to 12 years old with an average age of $5.12 \pm 2.26$ years old. Causes of burn injury: 20 cases of scald burns from hot water, 11 cases of flame burns, 3 cases burns of hot edible oil, and 3 cases burns of thermokalite (falling into lime pond). The burns areas from $25 \%$ to $92 \%$ TBSA (Deep degree burns from $10 \%$ to $72 \%$ TBSA) with an average of $37.2 \pm 3.2 \%$ TBSA.

\subsection{Data of Control Group (B Group)}

There were 32 cases with extensive and deep degree pediatric burns were treated by SGT and met the inclusion criteria. 20 males and 12 females, the age from 2 years old to 12 years old with an average age of $4.62 \pm 3.12$ years old. Causes of burn injury: 20 cases of scald burns from hot water or soup, 8 cases of flame burns, 2 cases of hot edible oil, and 2 cases of thermokalite (falling into lime pond). The burns areas from $25 \%$ to $85 \%$ TBSA (Deep degree burns from $10 \%$ to $60 \%$ TBSA) with an average of $34.2 \pm 2.9 \%$ TBSA.

\subsection{Comparability}

The age structure, degrees of burns, burns areas, causes of burns and the states of burns have no statistic difference $(\mathrm{P}>0.05)$ between A group and $\mathrm{B}$ group. The two groups have comparability.

\section{Methods}

\subsection{Anti-Shock and Systemic Treatment}

After admission, the treatment of anti-shock and fluid resuscitation was applied to both groups. A group accepted fluid resuscitation from $30 \mathrm{~min}$ to $2.5 \mathrm{~h}$ after burn injury ( 7 cases accepted fluid resuscitation before admission); B group accepted fluid resuscitation from $25 \mathrm{~min}$ to $2 \mathrm{~h}$ after burn injury (12 cases accepted fluid resuscitation before admission). The quantity of fluid resuscitation in the test group was calculated in accordance with the Fluid Resuscitation Formula proposed by Prof. $\mathrm{Xu}$ Rongxiang in Burns 
Regenerative Medicine and Therapy: \{The total amount of fluid required for resuscitation $(\mathrm{ml})=$ [amount of need for physiological fluid (ml) + TBSA $\%$ $\times$ body weight $(\mathrm{kg}) \times 1.0 \mathrm{ml}] \div[$ hourly urine volume (ml) $\div$ body weight in $\mathrm{kg}]\}$. The quantity of fluid resuscitation in the control group was calculated according to Children Fluid Resuscitation Formula proposed at The Chinese Burns Conference 1970, Shanghai, China (specialy for the skin-graft therapy): [The total amount of fluid required for resuscitation $(\mathrm{ml})=$ amount of need for physiological fluid $(\mathrm{ml})+$ TBSA $\% \times$ body weight in $\mathrm{kg} \times 1.5 \mathrm{ml}$. The liquids for fluid resuscitation include crystal solution, colloid solution and water (5\% or $10 \%$ Glucose) or mix-liquid of crystal and colloid (i.e., $400 \mathrm{ml}$ of Lactated Ringer Solution $+100 \mathrm{ml}$ of $20 \%$ Human Serum Albumin). Observe carefully the vital signs, urine volume of the children in the process of fluid resuscitation; adjust the quantity and speed of fluid resuscitation in terms of individual condition. Systemic treatments of anti-infection, protection and recovery of organ function and nutritional support in both groups were similar.

\subsection{The Wound Treatment in A Group}

For the lightly contaminated wounds, smear Moist Exposed Burns Ointment (MEBO) directly on the wounds; For the heavily contaminated wounds, adopt $0.1 \%$ bromogeramine and normal saline (NS) to clean the wounds before smear MEBO on the wounds, at the 1 st time only. The thickness of ointment on the wounds should be around $1 \mathrm{~mm}$ or cover the wound with a layer of prepared MEBO gauze directly, and adopt the semi-exposed or exposed treatments of BRT. The eschar of Deep II degree and III degree burns were treated by the thinning and incision method with tillage blade or "Humby" knife. In the course of wound treatment, according to the three "NO" principle which is No pain, No bleeding and No dryness, and the four "TIMELY" principle which is timely remove the necrotic tissues, timely remove the exudations, timely remove the liquefied products and timely remove the old MEBO apply new MEBO. During the eschar dissolving period (3-14 days after burns), clean the wound and change the dressing 3 4 times each day and then clean the wound and change the dressing 2 3 times per day, some routine non-narcotic analgesic (Rotundine, etc.) and some tranquilizer (Diazepam, Estazolam, etc.) can be applied only if the pediatric patient can not cooperate due to pain during dressing change. The large area III degree burns can close the wounds in combination with SGT or micro skin planting and cultivation technique if can not see the new skin islands over 42 days treatment of BRT. After wound healing, the patients are recommended to conduct physical exercise as well as to use elastic bandage, elastic vest or gloves for 3-12 months as clinical routine to prevent scar formation.

\subsection{The Wound Treatment in B Group}

Use $0.1 \%$ bromogeramine and NS to clean the wound at admission and before dressing change, then use iodophor to disinfect the wound and the skins surrounding the wounds followed by application of $\mathrm{SD}-\mathrm{Ag}$ to cover the wounds. For deep II degree wounds, use SD-Ag cold cream and treatment by bandage therapy, change the dressing every $1 \sim 2$ days. If there is eschar dissolving, clean the liquefied eschar gradually; for the wounds can not heal in 2 weeks, apply eshcar excision and skin grafting technique as the following treatment. For the III degree wounds, smear 1\% SD-Ag suspension on the wounds, dry the wounds with hair dryer, light or burns therapeutic apparatus, smear the SD-Ag 1 3 times per day. After the shock period and being in stable condition, use eschar excision and skin grafting technique (in parts for large area wounds) to close the wounds. After wound healing, similar to A group, the patients of group B are also recommended to conduct physical exercise as well as to use elastic bandage, elastic vest or gloves for 3-12 months as clinical routine to prevent scar formation. 


\subsection{The Standard of Evaluation}

(1) Pain during the treatment: judged pain according to the average using of analgesic during treatment period (The average using of analgesic $=$ the total times of analgesic using / the number of total cases); (2) The proliferation of scar is evaluated by the pliability and height of the scar in the follow-ups of 1st, 3rd, 6th and 12th month; (3) The diagnostic standard of wound infection[4]: fever $\left(\mathrm{T}>39^{\circ} \mathrm{C}\right)$, peripheral blood check $\left(\mathrm{WBC}>2.2 \times 10^{9} / \mathrm{L}\right)$, the condition of the wounds worsen, the unexpected rapid isolation of eschar and the appearance of empyema under the eschar, the surrounding skins of the wounds present the symptom of red, swollen, hot and pain, results of bacteria cultivation for blood and wound are positive; (4) Dysfunction means the joint loses more than $50 \%$ function or the moving angle becomes less than $50 \%$ of its original extent after treatment; (5) Analgesic used in this study are non-narcotic pain killer, including morphine, pethidine, fortanodyn, rotundine, Somiton, etc.

\section{Results}

The statistics of the data of all cases shows: In the test group, there is 1 case died (mortality is $2.7 \%$ ) which happened on the $9^{\text {th }}$ day and the cause of death was severe inhalation injury and respiratory failure; In the control group, there is one case died (mortality is
$3.13 \%)$ which happed on the 13th day and the cause of death was MRSA (methicillin-resistant Staphylococcus aureus) infection, sepsis and multiple organ failure. The detailed statistic data of other outcome measures refer to Table 1 and Table 2.

\section{Typical Cases}

\subsection{Test Group (BRT)}

Patient A: Male, 2 years and 6 months old. Cause of burns: boiled water scald. Clinical diagnosis: extensive ultra severe burns, TBSA $=80 \%$ (III degree burns $30 \%$, Deep II degree burns 30\%, Superficial II degree burns $20 \%$ ). The statuses of wound healing at different periods are shown in Figs. 1-8 below.

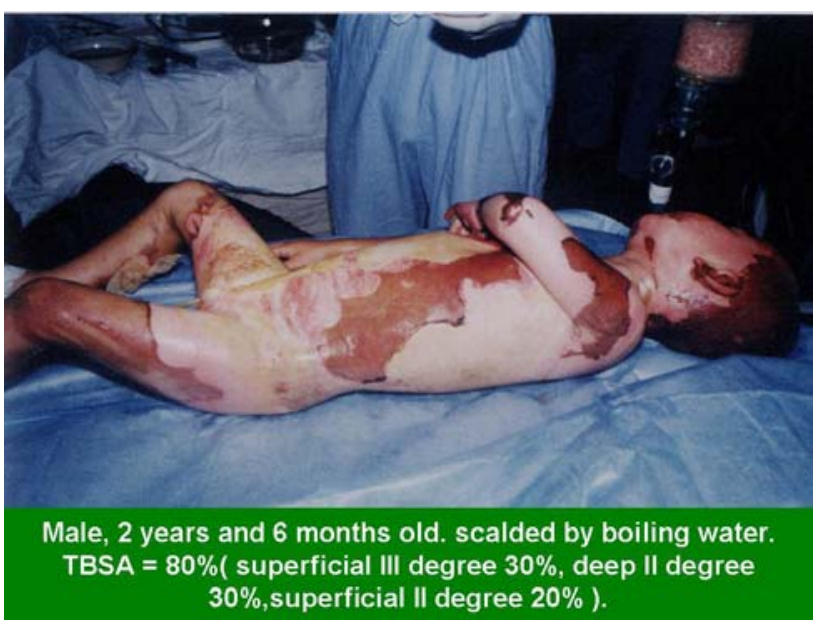

Fig. 1 The 4th days after burns (Shifted from other hospital).

Table 1 The comparison between two groups.

\begin{tabular}{cccccc}
\hline Group & Qty & Average using of analgesic ${ }^{1}$ (Times) & Infection (\%) & Skin-grafting (\%) & Wound healing time \\
\hline Group A & 37 & $5.75 \pm 2.61(2 \sim 11)^{*}$ & $24.32(9 / 37)^{* *}$ & $16.21(6 / 37)^{* * *}$ & $29.5 \pm 4.25(23 \mathrm{~d} \sim 67 \mathrm{~d})^{\#}$ \\
Group B & 32 & $11.50 \pm 3.24(6 \sim 21)^{*}$ & $34.38(11 / 32)^{* *}$ & $100(32 / 32)^{* * *}$ & $37.5 \pm 6.31(19 \mathrm{~d} \sim 89 \mathrm{~d})^{\#}$ \\
\hline
\end{tabular}

Average using of analgesic $=$ the total times of analgesic using / the number of total cases

Chi-square test between group A and group $\mathrm{B}:{ }^{*} \mathrm{X}_{1}{ }^{2}=35.49,{ }^{* *} \mathrm{X}_{2}{ }^{2}=10.72,{ }^{* * *} \mathrm{X}_{3}{ }^{2}=20.38$,

T check between group A and group $\mathrm{B}:{ }^{\#} \mathrm{t}=2.187, \mathrm{P}<0.01$,

There is significant difference between group A and group B.

Table 2 The comparison between two groups after wounds closing.

\begin{tabular}{cccc}
\hline Group & Qty & Scar hyperplasia (\%) & Dysfunction (\%) \\
\hline Group A & 37 & $32.43(12 / 37)^{*}$ & $8.11(3 / 37)^{* *}$ \\
Group B & 32 & $100(32 / 32)^{*}$ & $78.13(25 / 32)^{* *}$ \\
\hline
\end{tabular}

Chi-square test between group A and group $\mathrm{B}:{ }^{*} \mathrm{X}_{1}{ }^{2}=23.59,{ }^{* *} \mathrm{X}_{2}{ }^{2}=15.02, \mathrm{P}<0.01$,

There is significant difference between group A and group $\mathrm{B}$. 


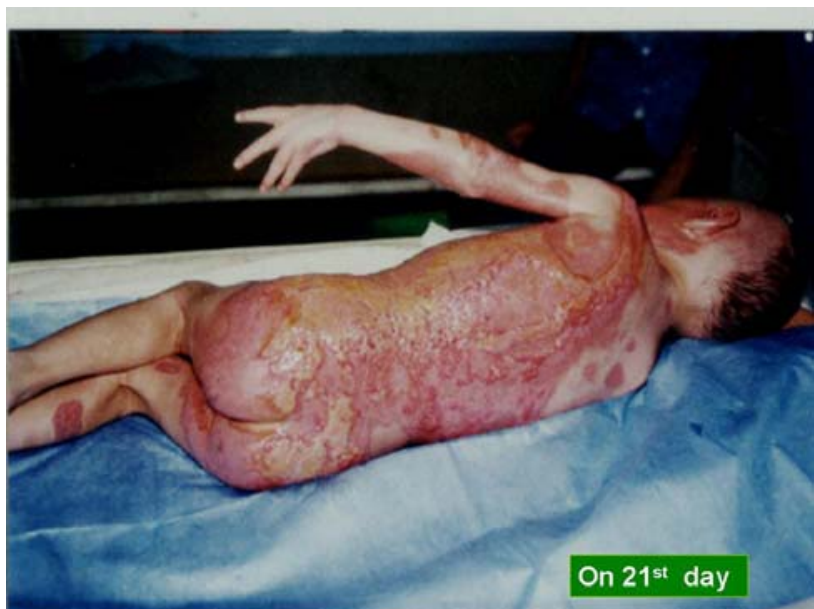

Fig. 2 The wounds on the back after 21 days' treatment (Deep II wounds healed).

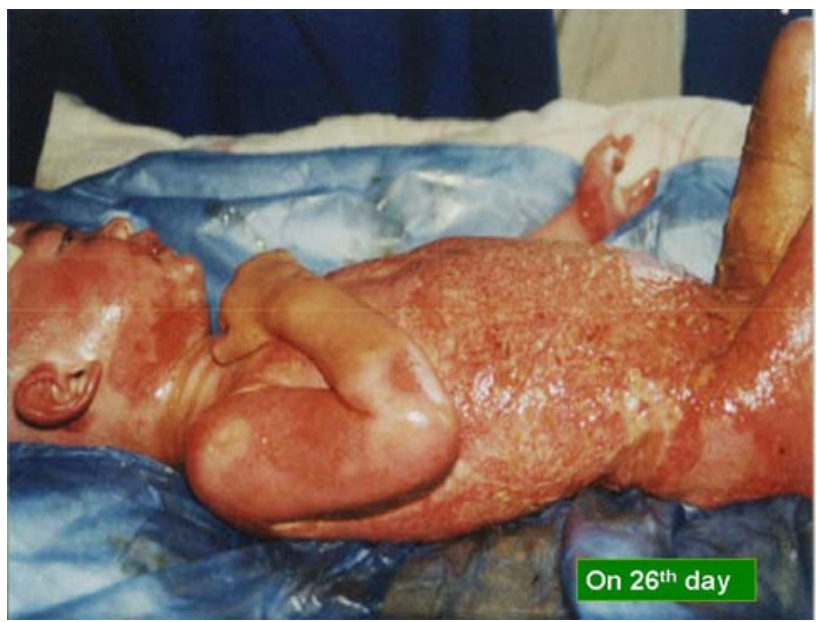

Fig. 3 Superficial III burn wounds on the chest and abdomen after 26 days' treatment.

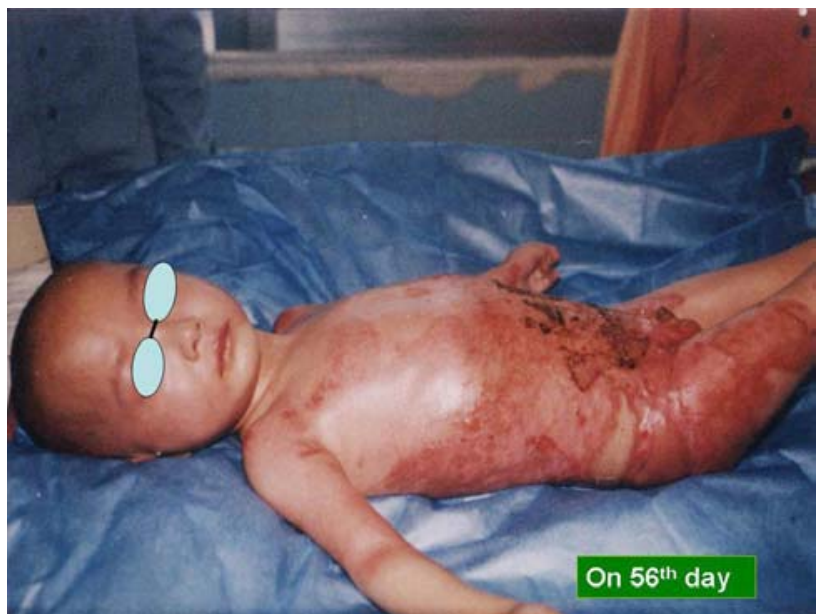

Fig. 4 The condition of wounds healing on 56th day.

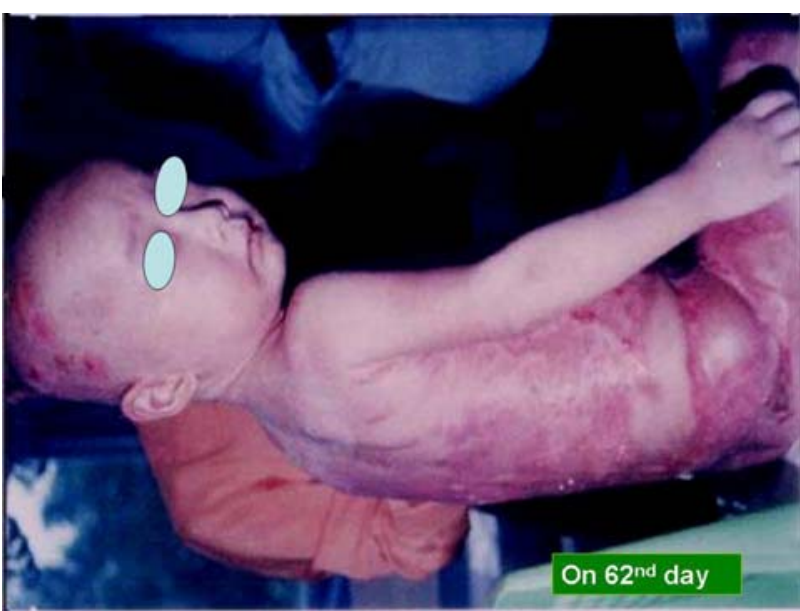

Fig. 5 The condition of wounds healing on 62nd day.

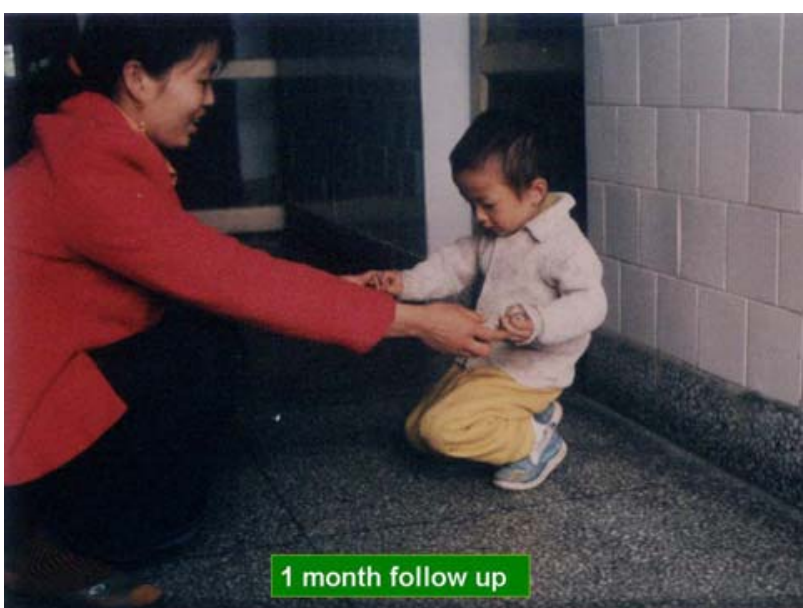

Fig. 6 Following-up after discharged from hospital 1 month later and the case recovered the function of walk and crouch.

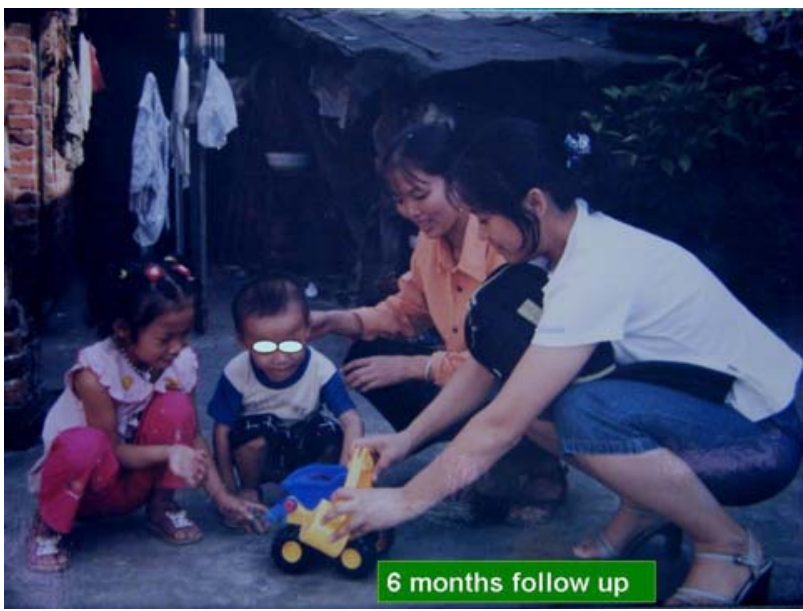

Fig. 7 Following-up after discharged from hospital 6 months later and the life of the case became normal. 


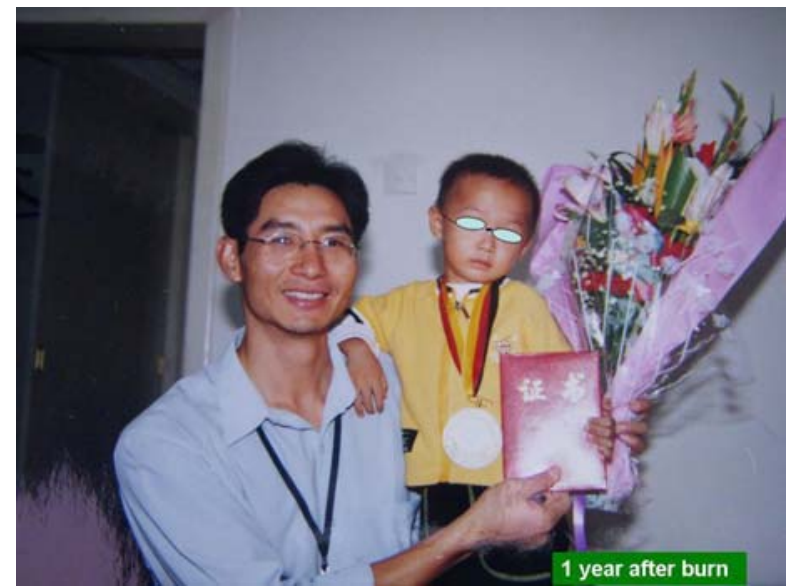

Fig. 8 Following-up after discharged from hospital 1 year later. No scar hyperplasia and disability appeared. The case received the certificate of The Fourth Burns Rehabilitation Stars.

Patient B: Female, 5 Years old. Cause of burns: Flame. Clinical diagnosis: large area and severe deep burns, $\mathrm{TBSA}=92 \%$ (III degree burns 26\%, Deep II degree burns $34 \%$, Superficial II degree burns 32\%) (Figs. 9-17).

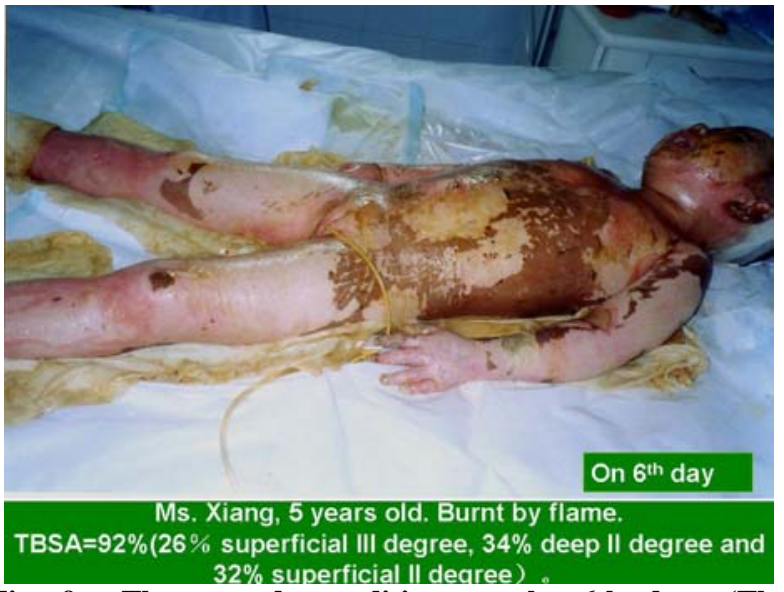

Fig. 9 The wounds condition on the 6th days (The liquefaction of the necrotic tissues).

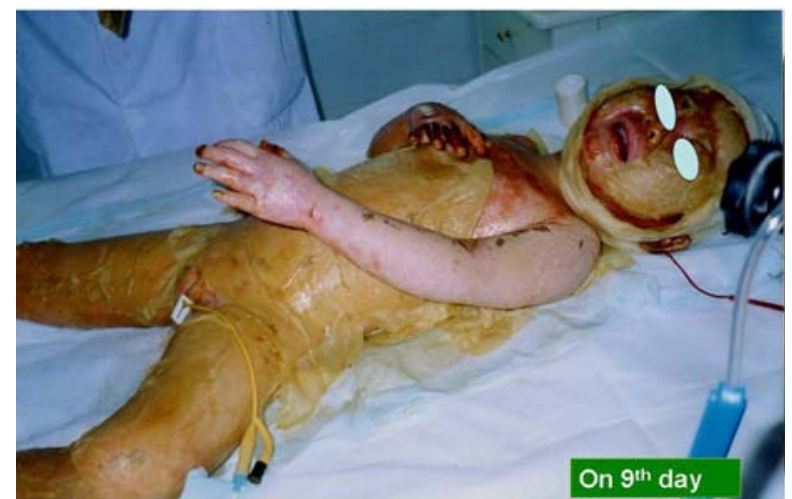

Fig. 10 The wounds condition on the 9th days (The liquefaction of the necrotic tissues continues).

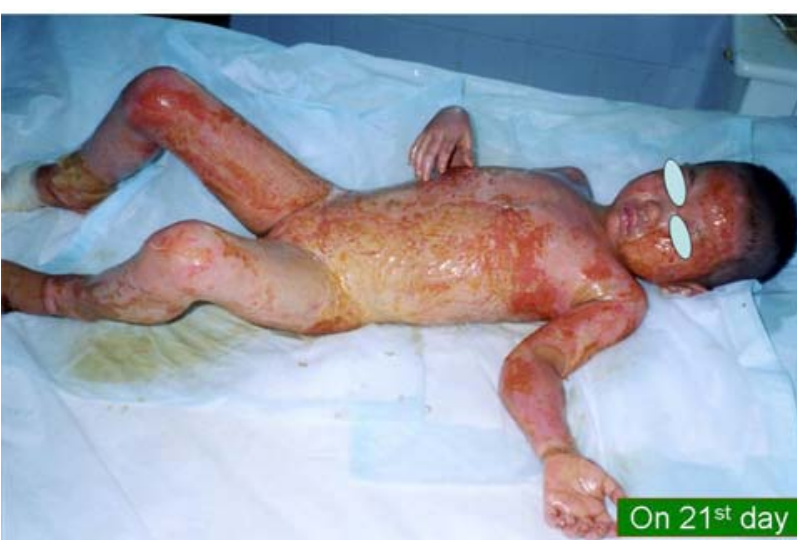

Fig. 11 The wounds condition on the 21st days (The necrotic tissues are all liquefied and cleaned).

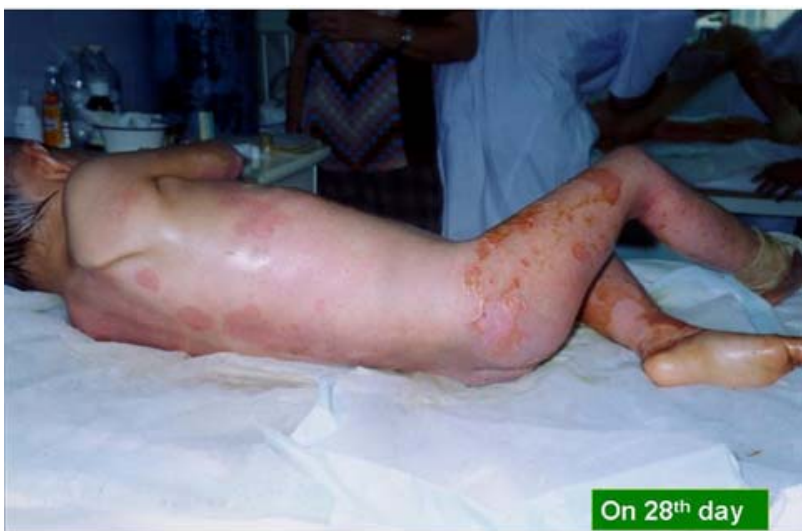

Fig. 12 The wounds condition on the 28th days (Deep II healed).

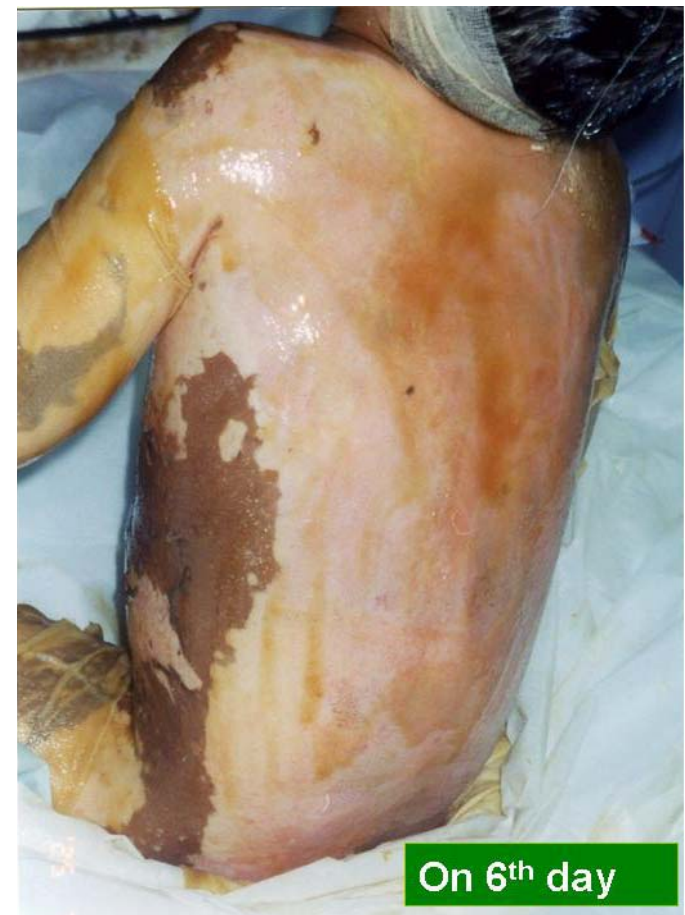

Fig. 13 The wounds condition on the back on the 6th day. 


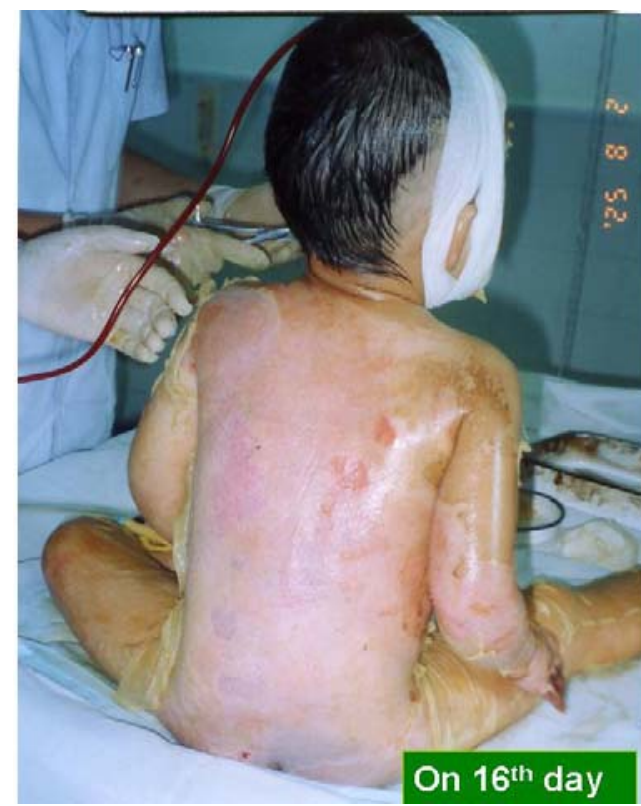

Fig. 14 The wounds condition on the back on the 16th day.

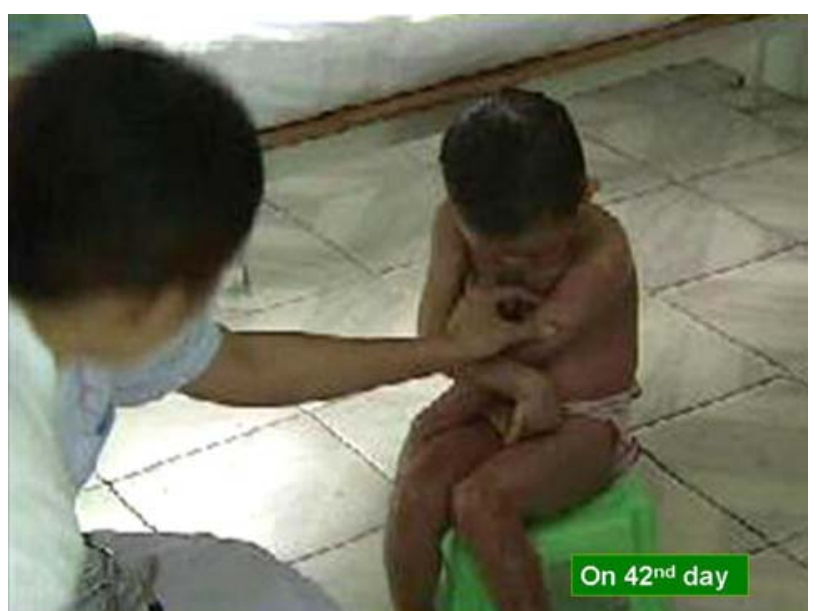

Fig. 15 All the wounds healed after 42 days' treatment and the skin elasticity is excellent.

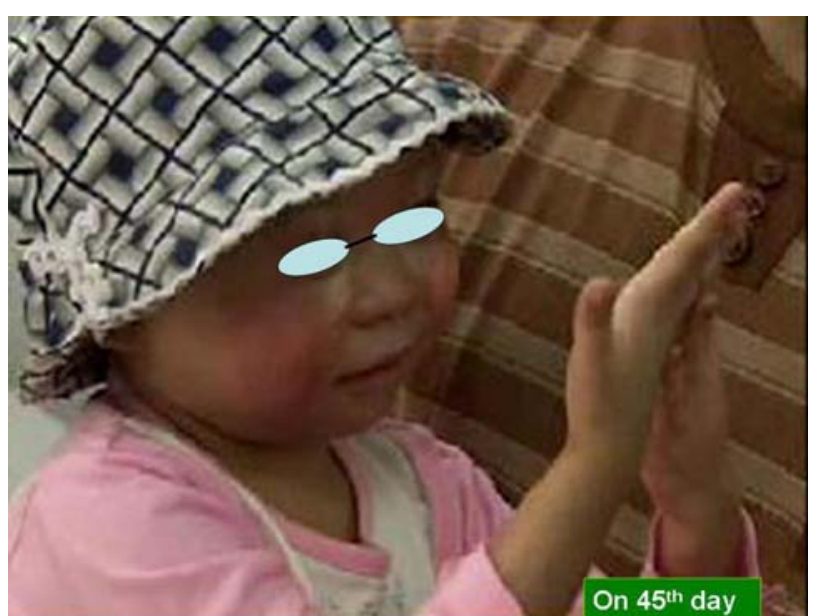

Fig. 16 The wounds on the head and hands recovered very well.

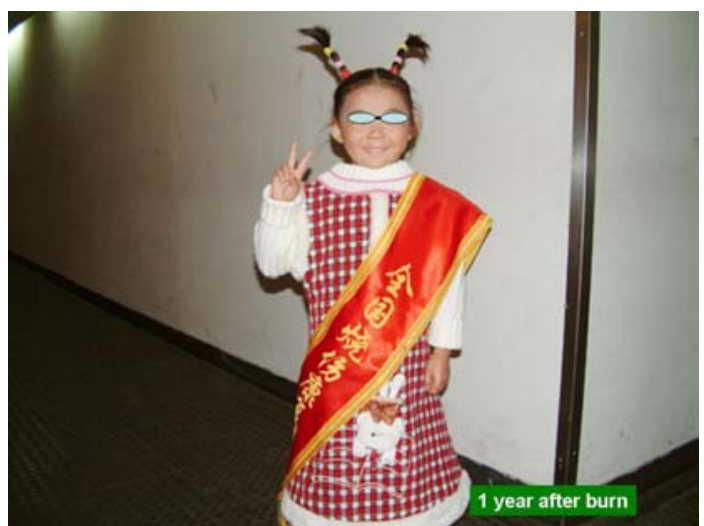

Fig. 17 Following-up after discharged from hospital 1 year later. No scar hyperplasia and disability appeared.

\subsection{Control Group (Eschar Excision and Skin-Grafting)}

Patient C: Male, 10 years old. Cause of burns: Hot water. Clinical diagnosis: large area and severe deep burns, TBSA $=46 \%$ (Superficial II degree burn $9 \%$, deep II degree burn 20\%, III degree burn 17\%). The statuses of wound healing at different periods are shown in Figs. 18-23 below.

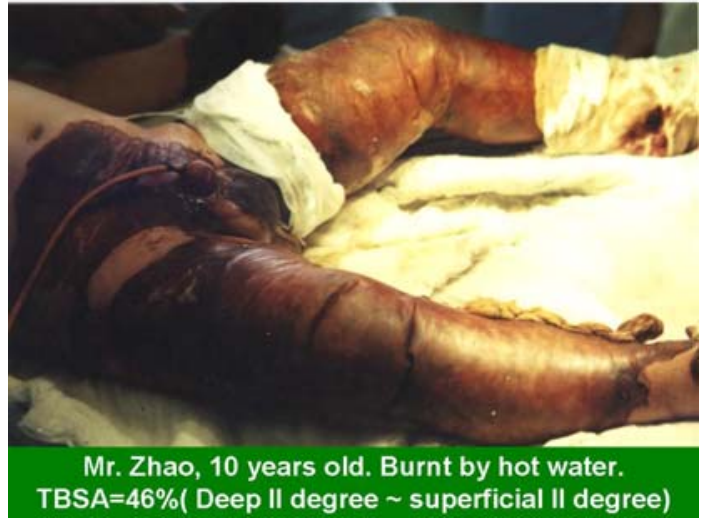

Fig. 18 Use disinfectant to clean the wounds after hospitalized (Dry the wounds and form the eschar).

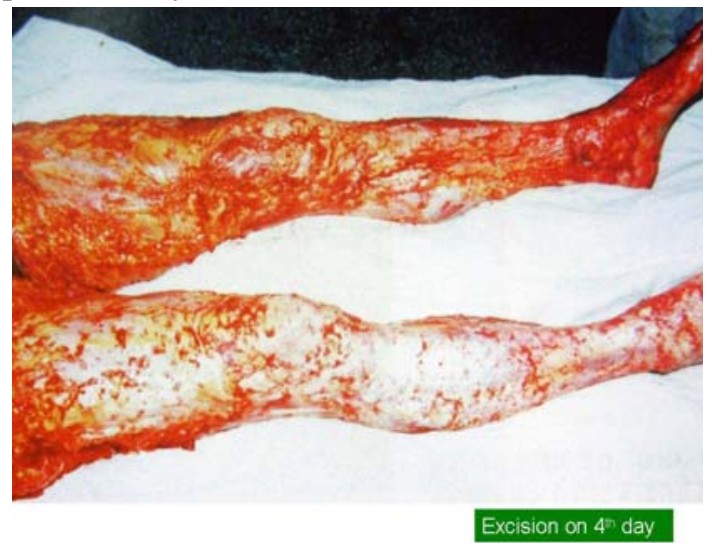

Fig. 194 days after hospitalized, use eschar excision on the lower limps. 


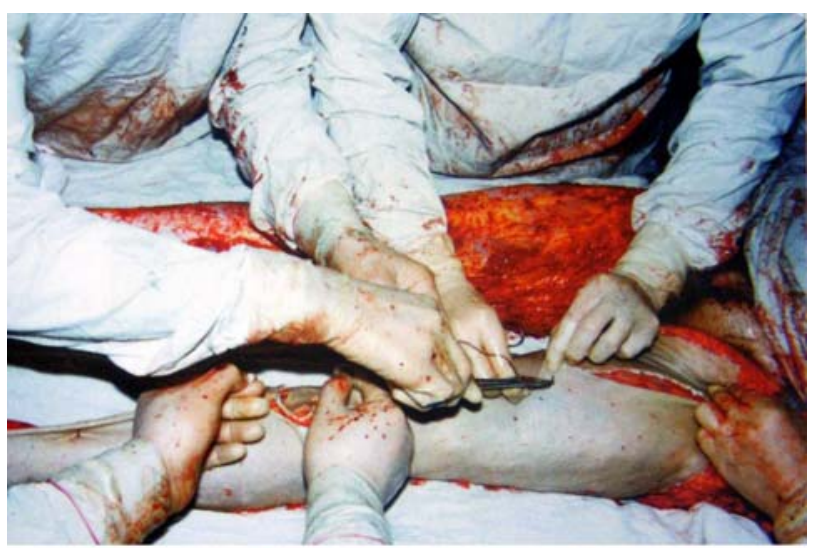

Micro-autograft combine with allograft on $4^{\text {th }}$ day

Fig. 20 Micro-autograft combined with allograft on 4th day.

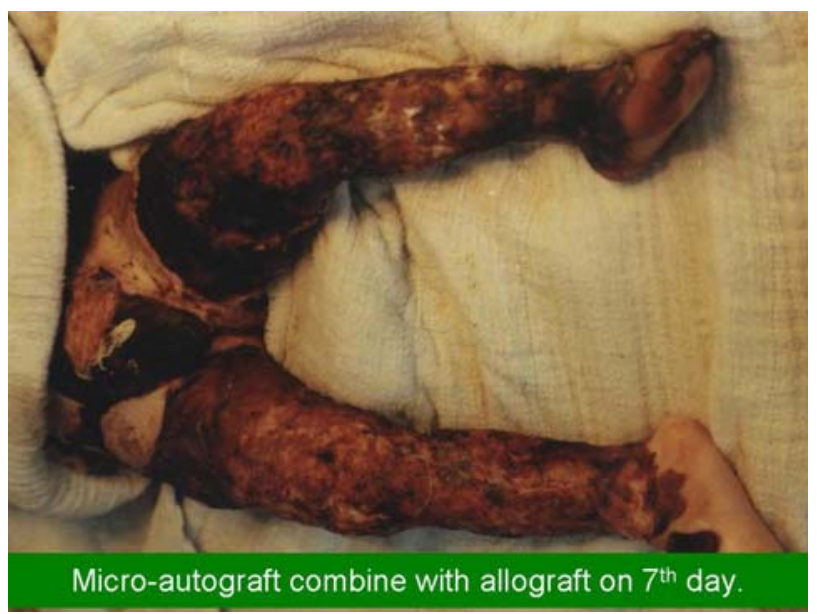

Fig. 21 Micro-autograft combined with allograft on 7th day.

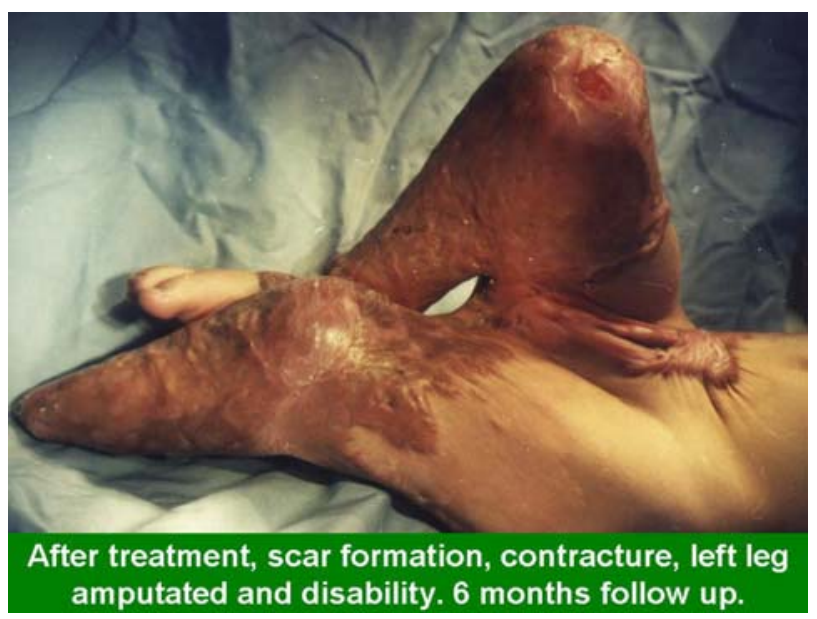

Fig. 22 Following-up after 6 month, scar formation, contracture and disability appear.

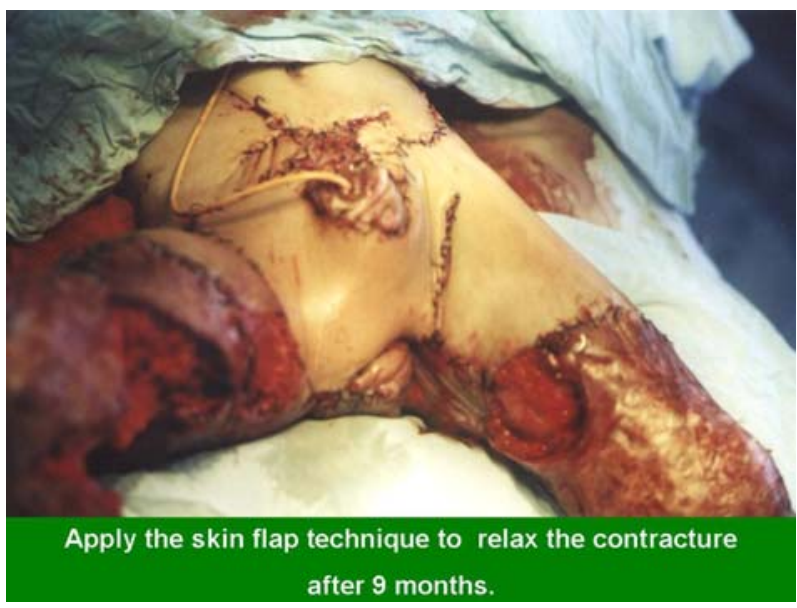

Fig. 23 Apply the scar relaxing and skin flap transplanting technique after 9 months.

Patient D: Male, 4 Years old. Cause of burns: Hot water. Clinical diagnosis: large area and severe deep burns, TBSA $=36 \%$ (Deep II degree burns-III degree burn) (Figs. 24-27).

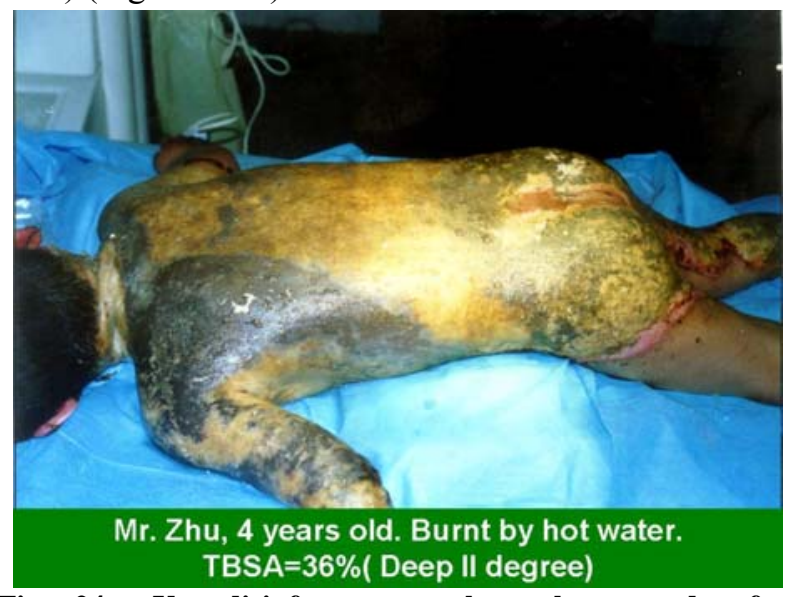

Fig. 24 Use disinfectant to clean the wounds after hospitalized (Dry the wounds and form the eschar).

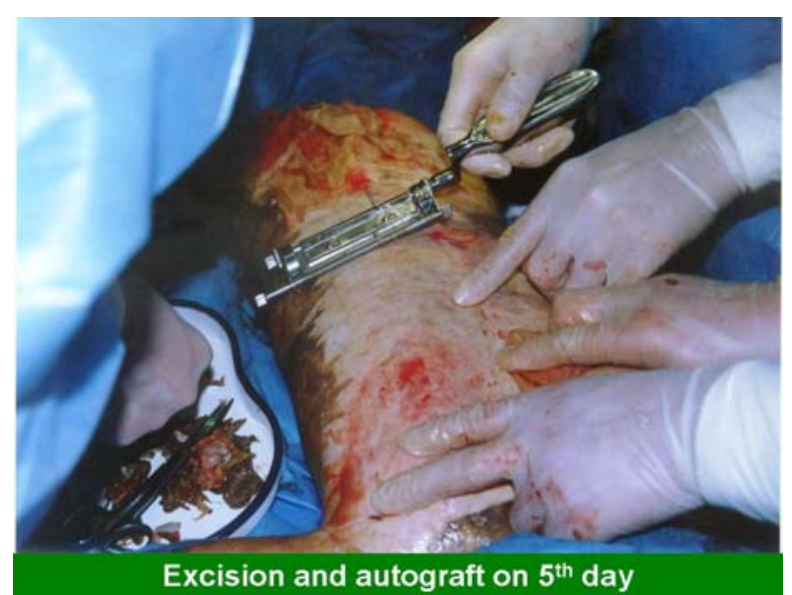

Fig. 255 days after hospitalized, apply the treatment of eschar excision and auto-grafting. 


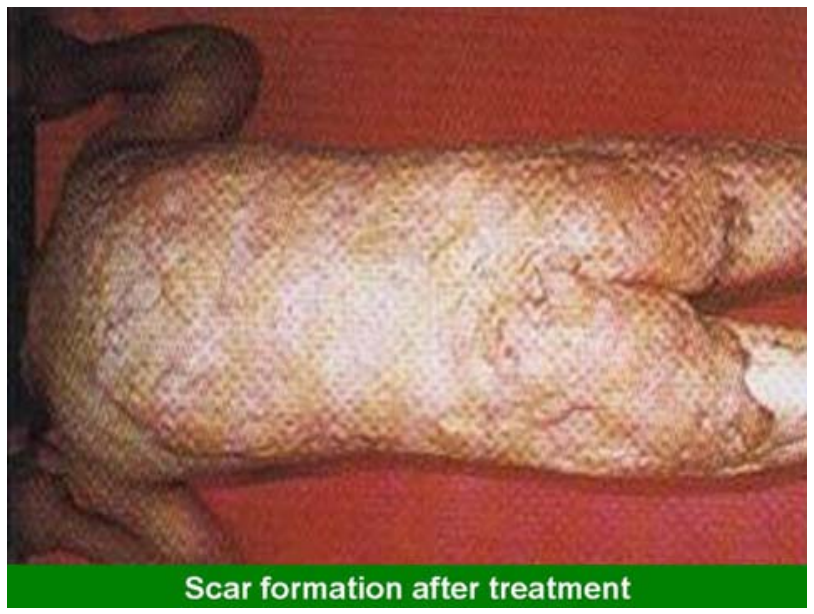

Fig. 26 Following-up one month later after discharged and there is apparent scar hyperplasia.

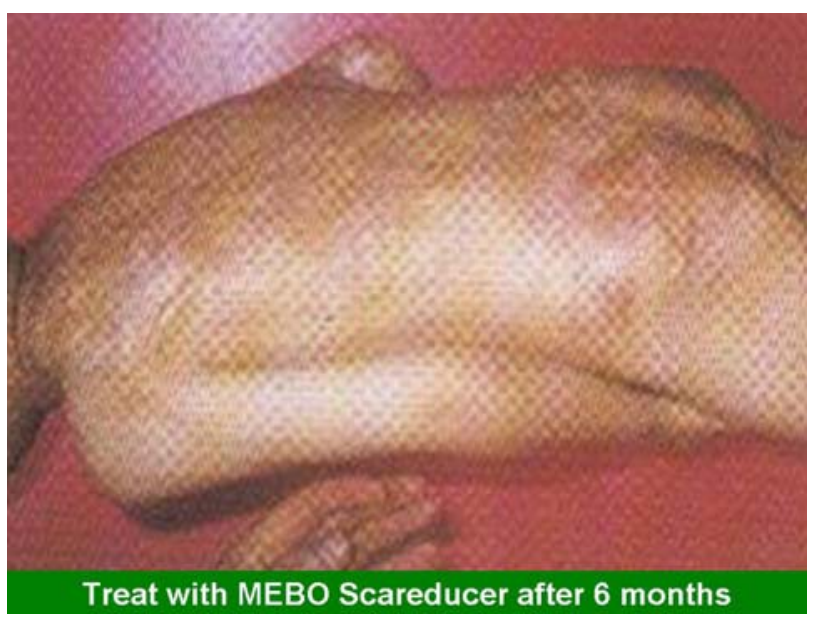

Fig. 27 After using MEBO Scareducer 6 months and the hyperplastic scars reduced.

\section{Discussion}

As we know, before the 2nd World War, medical doctors could do nothing but amputation to save the lives of the deep burn patients, because the wounds were always rotten and couldn't heal. It was Dr. Joseph E. Murray who tried excising the burnt skin and applied skin grafting to promote wound healing. He made great success on saving the patient's lives. Soon after that, the surgical skin graft technique for burn treatment was set up, which mainly included two parts: (1) Smear antibiotics or disinfectors on wound and keeping dryness for prevent the infection at early stage; (2) Apply eschar excision and auto-skin grafting technique to close the wound. Dr. Joseph E. Murray won the Nobel Prize due to the invention of skin graft and kidney transplantation in 1990 [5]. Since Dr. Joseph E. Murray invented the burns surgical eschar excision and skin grafting technique late 1940s, this technique has achieved a great improvement and development in the past 60 years. It has been great help to save patient's lives and lower mortality of burns. However, it is necessary and well worth considering how to treat the burns patients so that they can restore and return to routine life more effectively [6]. For the technology of eschar excision and skin grafting, pain, infection, secondary injury of wounds, scar hyperplasia and dysfunction in and after treatment are still remained as unsolved important problems [7]. In addition, it is more difficult to treat the deep burns of children than adults. Once happened, the pediatric burns are usually deep degree because children's skin is thin, soft and tender with thinner stratum corneum, the basilar membrane between the epidermis and dermis is imperfect, and the appendages of skin are undeveloped. The conventional method of applying the disinfectant, antibiotics and baking on the wounds can dry the wounds to form dehydrated eschar and result in vascular spasm and contracture, followed by the necrosis of the stasis zone due to dehydration, ischemia, hypoxia, and devitalization, which in turn deepen the wound into hard to heal III degree wound [8]. It is very difficult to avoid damage on the alive tissues, and the subcutaneous tissue be remove usually when we do eschar excision on the burnt wound. We need to do several times skin grafting in order to close the extensive deep degree burn wound, and duration is longer. Therefore the conventional method can increase the possibility of secondary injury and scar hyperplasia after skin graft, and the excessive scars will impact the growth and development of children. Moreover, children have low compliance in treatment due to weak tolerance for pain. So the main purpose of the treatment of deep pediatric burns are to alleviate farthest the pain, control the infection, avoid deepening the wounds, shorten the healing time, improve the healing quality, and reduce the scar proliferation and 
contracture. With proper and timely treatment, the pediatric burns heal faster than adults, so the method having little stimulation, high speed of healing and low incidence of scar proliferation should be applied. However, most drugs used on the wounds are disinfectant and antibiotics which have not ideal outcome in treatment of deep pediatric burns. The conventional eschar excision and skin grafting technique mainly adopts the SD-Ag or SD-Zn as drugs used on wounds. The SD absorbed through wound bed can affect the metabolism of bilirubin [9] and the absorption of silver ions contained in SD-Ag was reported to cause silver poisoning [10].

Burns regenerative medicine and therapy (BRT) is a fire-new technique for burns treatment which aims at physiopathology of burns in accordance with life rule, with the core technique of in situ skin regeneration at wound and criteria of healing without scar proliferation for deep burns. The clinical research of Keratin-19 (K-19) positive stem cells in situ regeneration of full-thickness burnt skin [11] using "Biotin-Antibiotin protein DCS immunofluorescence" method has confirmed that some of the human somatic cells (the potential regenerative cells, PRCs) can be activated and induced into K-19 positive stem cells, which in turn proliferate and differentiate to regenerate and form the new skin in the full-thickness burnt wound. The mechanism of BRT achieve skin regeneration including: (1) To liquefy and discharge the necrotic tissues without further damage; (2) To create a physiological moist environment on the wound; (3) To activate and translate the potential regenerative cells (PRCs) to stem cells and cultivate the stem cells and tissues in situ, achieve the physiological wound healing in the way of in situ regeneration and restoration [12]. This technique is achieved by the combination of Moist Exposed Burn Therapy and Moist Exposed Burn Ointment (MEBT/MEBO). MEBO, the drug used on wounds in BRT, has the following prominent effects and characteristics: (1) Pain alleviation: Isolate the wounds from the external environment and form a protective membrane which can protect the nerve endings, the berberine contained in the ointment can alleviate the spasm of arrectors pilorum muscle and baicalin can relieve microvascular spasm which relieve the pain of burns (achieved usually 1 5 minutes after application of the drug); (2) Avoid the progressive deepening of the wound: thanks to the melting of the unique frame structure of the ointment dosage form, it will absorb the residual heat of the wounds and relieve the continued physical heat injuries; (3) Liquefy and discharge necrotic tissues without further damage to surrounding tissues through a series of biochemical reactions including hydrolysis, enzymolysis, rancidity, saponification, lipidation and esterification; (4) Prevent and control infection through the following means: control the bacteria density at wound, change the bacteria morphology, reduce the bacteria proliferation and decrease the bacteria toxin [13] with the help of effective ingredients of the drug and timely active drainage. By the standard of wound management, we clean the wound and change dresssing 3-4 times per day, and early to incise and thin the eschar of the deep wounds, to open the wound and get good drainage for the burnt toxin, necrotic tissue, liquefied product, bacteria and exudate easy to move outside, then achieve prevent and control the invasive infection; (5) Effective anti-inflammatory and anti-toxic functions derived from the $\beta$-sitosterol, baicalin, berberine and other active ingredients in the ointment; (6) In the physiological moist environment, the signal of injury, stimulation of inflammation, and regenerative substances in the drug activate and initiate the transformation of stem cells from the potential regenerative cells deep seated in the wounds. With the cultivation of rich nutrition in the drug, the stem cells differentiate, proliferate and connect continually to form new skin and physiologically restore the injured skin; (7) The wound can physiologically heal without scar proliferation. The ratio imbalance of epithelial tissue and fibrous tissue and the abnormal arrangement of collagen fibrous and capillaries are the main reasons 
of scar proliferation [14]. In the physiological moist environment and with the support of rich life regenerative substances and nutrients, various types of cells can grow in proportion and the fiber cell proliferation can be inhibited. So the injured skin can physiologically heal without scar proliferation [15].

In comparison with conventional surgical skin grafting therapy in treating extensive deep pediatric burns, BRT can effectively alleviate the pain, reduce infection and accelerate healing of the burnt wound. Meanwhile, BRT also can save farthest the alive tissues under necrotic tissues, realize the in situ skin regenerative healing, reduce the scar proliferation, lower or avoid the necessity for skin grafting, decrease the new surgical injury and risk of operation, shorten the course of treatment, decrease the treatment cost, make deep II degree wounds heal with no or little scar proliferation. According to the statistic analysis of medical data and the result of comparative study, BRT was proved to have certain superiority and worth spreading in the treatment of extensive deep pediatric burns. Further research is required to improve BRT and clarify how to promptly remove the necrotic tissues and alleviate the damage of tissues and organs due to burn toxins and inflammatory mediators and the resulted apparent fever reaction.

\section{Abbreviations:}

Burns Regenerative Medicine and Therapy $=$ BRT .

Skin Grafting Techniques $=$ SGT .

Moist Exposed Burns Ointment = MEBO.

\section{References}

[1] H. Xiao, T. Xie, L. Lin, S. H. Liu, J. Wu and T. R. Fang, Analysis on the burns reasons of 17339 cases, Chinese Journal of Epidemiology 21 (5) (2000) 333-334.
[2] R. X. Xu, The Blue Book Of Burns Regenerative Medicine, Vol. 1, Beijing: China Medical Science and Technology Press, 2000.

[3] A. Li, Burns Therapeutics (2nd ed.), Beijing: People's Medical Publishing House, 1995.

[4] R. X. Xu, Clinical Handbook of Burn Skin Regenerative Technique, Beijing: China Medical Science and Technology Press, 2000, p. 22.

[5] Baidu News, The Nobel Prize in Physiology or Medicine, 1990, p. 5.

[6] D. N. Herndon, Total Burn Care (1st ed.), America: Saunders, 2004.

[7] R. X. Xu, Chinese Burns Wounds and Ulcer, Beijing: Chinese Technology Center for and Burns Wounds and Ulcer, 1997.

[8] B. Zeng and S. H. Yao, Clinical Experience with MEBT/MEBO in Treating Neonatal Burn, Chinese Journal of Burns Wounds \& Surface Ulcers 13 (3) (2001) 183-184.

[9] X. Y. Chen, Y. Li and D. L. Hu, Clinical experience in treating neonatal burns, Chinese Journal of Burns Wounds \& Surface Ulcers 13(3) (2001) 181-182.

[10] J. R. Shi and Y. L. Chen, A case report of sulfadiazine silver in treating burn wound that lead to subacute argyria, Chinese Journal of Plastic Surgery 4 (3) (1988)228-229.

[11] R. X. Xu, The Burns Regenerative Medicine and Therapy, Switzerland: Karger; 2004.

[12] R. X. Xu and M. Xiao, The mechanism of burn regenerative therapy and wound healing, Chinese Journal of Burns Wounds \& Surface Ulcers 15 (4) (2003) 253-271.

[13] R. X. Xu, Clinical handbook of burn regenerative therapy and medicine, Beijing: China Medical Science and Technology Press, 2003.

[14] Z. D. Chang, The Reparation and Systemic Treatment on Burns Wounds. Beijing: Beijing Publishing House; 1993.

[15] R. X. Xu, Burn regenerative therapy, Chinese Journal of Burns Wounds \& Surface Ulcers 9 (3) (1997) 4-12. 